\title{
DEVELOPMENT OF A NUMERICAL METHOD FOR SOLVING THE INVERSE CAUCHY PROBLEM FOR THE HEAT EQUATION
}

\author{
(C) 2019 H.K. Al-Mahdawi \\ South Ural State University \\ (pr. Lenina 76, Chelyabinsk, 454080 Russia) \\ E-mail: hssnkd@gmail.com
}

Received: 04.06.2018

In this work, the initial temperature has been investigated in the Cauchy inverse problem for linear heat conduction equation that it depends on the given temperature at specification time. In this problem, the initial temperature distribution is unknown, but instead, there is a known temperature at the time, $\mathrm{t}=\mathrm{T}>0$. The heat conduction problem can be formulated as Fredholm integral first kind equation. It is well known that this problem is an ill-posed problem and direct solution to this problem is unacceptable. An algorithm has been used to define a finite-dimensional operator for this problem also used the generalized discrepancy method to reduce the conditional extremum variation problem to unconditional extremum variation problem for the integral equation. The discretization of the integral equation has made it possible to reduce this problem to a system of linear algebraic equations. Then, Tikhonov's regularization inversion method has been used to find an approximation solution. Finally, the numerical computation example has been presented to verify the accuracy of the estimated solution.

Keywords: ill-posed problem, regularization, inverse problem, heat conduction.

\section{FOR CITATION}

Al-Mahdawi H.K. Development of a Numerical Method for Solving the Inverse Cauchy Problem for the Heat Equation. Bulletin of the South Ural State University. Series: Computational Mathematics and Software Engineering. 2019. vol. 8, no. 2. pp. 22-31. DOI: $10.14529 / \mathrm{cmse} 190202$.

\section{Introduction}

The Cauchy inverse problem of heat equation is ill-posed in the sense that arbitrarily "small" change in the data can produce "large" errors in the solution. The problem can be defined in the sense of Jacques Hadamard, that a problem is well-posed if and only if the following properties hold [1].

- The solution exists, at least one solution exists (existence).

- The solution is unique, at most one solution exists (uniqueness).

- The solution depends continuously on the data (stability).

It is impossible to solve the ill-posedness problem by using classical numerical methods. It requires special techniques, e.g., regularization strategies. With the development of high-speed personal computers, it has become more convenient to use numerical techniques to solve heat transfer inverse problems. Theoretical concepts and computational implementation related to Cauchy inverse problem of heat equation have been discussed by many authors, and a lot of methods have been described [3-7].

In some of them, the author has been identified the heat flux at the front surface of a thick plate based on the measured temperature history at the plate back surface, which is insulated [3]. In [4] the author has been applied the numerical method involving the Laplace transform technique and the finite difference method in conjunction with the least-squares 
scheme to an Inverse Heat Conduction Problems. The inversion model that simultaneously highlights the measurement errors and the inaccurate properties of the forward problem has been proposed in [5] to improve the inversion accuracy and robustness. With the assistance of the Tikhonov regularization method, a cost function is constructed to convert the original an Inverse Heat Conduction Problems into an optimization problem [5]. In other paper, a model has been developed to solve the inverse heat conduction problems for a triangular wall. The conjugate gradient method has been used with the finite element method to determine the twodimensional variations of the temperatures and heat fluxes on the wall surface with time [6]. In [7] the Cauchy problem for the Laplace equation in a multiply connected region was solved by replaced the heat conduction problem to the Poisson equation and solve it in a simply connected region with an unknown source function different from zero in the adjoined region. The methods described in the [2] are used to solve a number of inverse problems in mathematical physics. The fundamentals of the optimal methods have been obtained for solving ill-posed problems, as well as ways to estimate accurate solution and accurate by order error estimates for these methods.

The main idea in this paper is to reconstruct the source function of the diffusion equation by using the algorithm which proposed in [8]. The corresponding inverse problem, by Fourier series expansion, has been represented as Fredholm equation of the first kind. Hence, the solution does not depend continuously on the data in conventional Banach spaces, so the solution unstable $[1,2,8]$. Therefore, this is an ill-posed problem. To get a well-posed problem Tikhonov Regularization will be using. The problem of selecting the best regularization parameter will be solved in this paper by using the residual principal method which described in [2].

All these steps will be implemented through the sections in this paper. Section 1 defines the direct problem for heat conduction problem as a linear partial differential equation and describes solution as an integral Fredholm equation of the first kind. Section 2 defines the inverse problem and give a discerption about the known data and operator. Section 3 considers the integral equation of the first kind and reduces it as a system of linear algebraic equations by implementing the algorithm in [8]. Then, in Section 4 the example has been presented to verify the accuracy of our estimated solution. Finally, the explanation of the suggested method has been summarized in the conclusion Section with suggested future work for solving the nonlinear backward heat problem.

\section{Direct problem}

The direct (forward) problem consists of passing heat conduction through a bar with the determined boundary condition and initial temperature condition. The mathematical formulation of this problem has been described by the following liner partial differential equation

$$
\begin{gathered}
\frac{\partial u(x, t)}{\partial t}=D \frac{\partial^{2} u(x, t)}{\partial x^{2}}, 0<x<1, t \geq 0, \\
u(0, t)=0, t \geq 0 \\
u(l, t)=0, t \geq 0, \\
u(x, 0)=u_{0}(x), 0 \leq x \leq 1,
\end{gathered}
$$


where the $u(0, t)$ and $u(1, t)$ are boundary conditions, $u_{0}(x)$ initial condition it is representing the initial temperature. The $(\mathrm{t})$ represents the time, $(\mathrm{x})$ spatial variable and $(\mathrm{D})$ denote the dispersion coefficient.

In the direct problem (1-4), the initial condition has been specified. For solving this type of problem there are many ways such as finite different method (FMD) and separation of variables. To formulate this problem as the Fredholm integral equation first kind, the Fourier series method by separation of variables has been used as follows:

$$
\begin{aligned}
& u(x, t)=\sum_{n=1}^{\infty} a_{n} e^{-(n \pi)^{2} t} \sin (n \pi x), \\
& u(x, 0)=u_{0}(x)=\sum_{n=1}^{\infty} a_{n} \sin (n \pi x), \\
& \forall a_{n}=2 \int_{0}^{1} u_{0}(x) \sin (n \pi x) d x
\end{aligned}
$$

from (5) and (7) we get

$$
u(x, t)=2 \int_{0}^{1} \sum_{n=1}^{\infty} e^{-(n \pi)^{2} t} \sin (n \pi x) \sin (n \pi y) u_{0}(y) d y .
$$

The formula (8) is rewriting as integral equation first kind for some fixed $t=T$ as following:

$$
u(x, t)=\int_{0}^{1} K(x, y) u_{0}(y) d y, 0 \leq x \leq 1,
$$

or we can write it as following:

$$
A u(x)=\int_{0}^{1} K(x, y) u_{0}(y) d y
$$

where $K(x, y)=\frac{2}{l} \sum_{n=1}^{\infty} e^{-(n \pi)^{2} T} \sin (n \pi x) \sin (n \pi y)$ and $u_{0}(y)$ the initial function.

Where the kernel $K(x, y) \in C([0,1] X[0,1]), u_{0}(y) \in L_{2}[0,1]$ and $f(x) \in L_{2}[0,1]$. The kernel of the operator $A$ is closed.

\section{Inverse problem}

The inverse problem, described as the initial temperature $u_{0}(y)$ is the unknown function inside integral. To estimate the unknown initial temperature the measurement temperature has been given at specific time $\mathrm{T}$ over the specified space interval $0 \leq x \leq 1$

$$
u(x, T)=f(x), T>0 .
$$

The measurement temperature includes some noise $f_{\delta}(x)$, where $\delta>0$ defined as the range of error, $\left\|f_{\delta}(x)-f_{0}(x)\right\|_{L_{2}} \leq \delta$. Additionally, the inverse operator $A^{-1}$ is unbounded $\left\|A^{-1}\right\|=$ $\infty$, it means the solution usually poor approximated even small value of $\delta$. All this lead to the inverse heat conduction problem it ill-posed problem because the solution is not stable.

\section{Computational scheme}

We considered the following integral equation of the first kind. Our target reduces this problem to a system of linear algebraic equations

$$
A u(x)=\int_{0}^{1} K(x, y) u_{0}(y) d y=f(x), T>0 .
$$

The kernel $K(x, y, T)$ is an infinite series and we cannot handle infinite sum and when $n$ goes to $\infty$ the value $e^{-(n \pi)^{2} T}$ become very small for simplicity, we finite the sum of series to 10 times 


$$
K(x, y)=2 \sum_{n=1}^{10} e^{-(n \pi)^{2} T} \sin (n \pi x) \sin (n \pi y), T>0 .
$$

Now introduce the operator $\mathrm{C}$ map $L_{2}[0,1]$ into $L_{2}(0, T]$

$$
C u(y)=\int_{0}^{1} K(x, y) u(y) d y
$$

for the numerical solution of the equation (14) replace operator $\mathrm{C}$ by the finite-dimensional operator $C_{m}$, where $C \rightarrow C_{m}$ and $C_{m} \sim A$.

Next step need to divide interval $[0,1]$ into $m$ equal parts by points $x_{i}=\frac{i(1-0)}{m}, i=$ $0,1, \ldots, m-1$ and $y_{j}=\frac{j(1-0)}{m}, j=0,1, \ldots, m-1$, the width for each interval $h=\Delta x=\Delta y=$ $\left(x_{i+1}-x_{i}\right)$ and $x_{i}=y_{j}$.

Now introduce the kernel function

$$
K(x, y,)=\bar{K}\left(x_{i}, y_{j}\right)
$$

where $x_{i} \leq x<x_{i+1}, y_{j} \leq y<y_{j+1}, i=0,1, \ldots, m-1$ and $j=0,1, \ldots, m-1$

$$
C_{m} u(y)=\int_{0}^{1} \bar{K}\left(x_{i}, y_{j}\right) u(y) d y=f_{\delta}(x)
$$

where $u(y) \rightarrow\left(u_{j}\right), j=0,1, \ldots, m-1 . u_{j}=u\left(y_{j}\right)$ and $f_{\delta}(x) \rightarrow\left(f_{i}^{\delta}\right), i=0,1, \ldots, m-1, f_{i}^{\delta}=$ $f_{\delta}\left(x_{i}\right)$.

Now introduces the finite-dimensional subspaces $Y_{m}$ and $X_{m}$ of the space $L_{2}[0,1]$ consisting all functions on intervals $\left(x_{i}, x_{i+1}\right], i=0,1, \ldots, m-1,\left(y_{j}, y_{j+1}\right], j=0,1, \ldots, m-1$.

We denote by $P_{m}$ and $Q_{m}$ the metric projection operators from $L_{2}[0,1]$ onto $Y_{m}$ and $X_{m}$ subspaces respectively

$$
C_{m} u\left(y_{j}\right)=\int_{0}^{1} \bar{K}\left(x_{i}, y_{j}\right) u\left(y_{j}\right) d y=f_{\delta}\left(x_{i}\right) .
$$

By using the generalized discrepancy method which has been described in [8] for the approximate solution of equation (16). We will reduce the equation to the conditional extremum variation problem

$$
\inf \left\{\|u(y)\|^{2}: u(y) \in Y_{m},\left\|C_{m} u(y)+f_{\delta}{ }^{m}(x)\right\| \leq \delta^{2}\right\},
$$

where $f_{\delta}{ }^{m}(x)=Q_{m}\left[f_{\delta}(x)\right]$.

The variation problem (18) is reduced to unconditional extremum variation problem

$$
\inf \left\{\left\|C_{m} u(y)-f_{\delta}^{m}(x)\right\|^{2}-\alpha\|u(y)\|^{2}: u(y) \in Y_{m}\right\}, \alpha>0,
$$

which is the version of the Tikhonov regularization method

$$
C_{m} u_{j}=h \sum_{j=0}^{m-1} \bar{K}\left(x_{i}, y_{j}\right) u\left(y_{j}\right)=f_{i},
$$

where

$$
u_{j}=P_{m}\left[u\left(y_{j}\right)\right]=h \int_{y_{j}}^{y_{j+1}} u(y), j=0,1, \ldots, m-1,
$$

the form of operator $Q_{m}$ implies that $f_{\delta}{ }^{m}(x)=\left\{f_{i}: x_{i} \leq x<x_{i+1}, i=0,1, \ldots, m-1\right\}$, where

$$
f_{i}=Q_{m}\left[f_{\delta}\left(x_{i}\right)\right]=h \int_{x_{i}}^{x_{i+1}} f_{\delta}(x), i=0,1, \ldots, m-1 .
$$

From (19-22) and to give the approximate solution to $u(x)$, we can rewrite the problem as linear algebraic equations 


$$
h *\left[\begin{array}{clll}
\bar{C} & C_{m}\left(u_{j}\right)=f_{i} \\
\bar{K}\left(x_{0}, y_{0}\right) & \bar{K}\left(x_{0}, y_{1}\right) & \ldots & \bar{K}\left(x_{0}, y_{m-1}\right) \\
\bar{K}\left(x_{1}, y_{0}\right) & \bar{K}\left(x_{1}, y_{1}\right) & \ldots & \bar{K}\left(x_{1}, y_{m-1}\right) \\
\vdots & \vdots & \ldots & \vdots \\
\bar{K}\left(x_{m-1}, y_{0}\right) & \bar{K}\left(x_{m-1}, y_{1}\right) & \ldots & \bar{K}\left(x_{m-1}, y_{m-1}\right)
\end{array}\right]\left[\begin{array}{c}
u\left(y_{0}\right) \\
u\left(y_{2}\right) \\
\vdots \\
u\left(y_{m-1}\right)
\end{array}\right]=\left[\begin{array}{c}
f_{\delta}\left(x_{0}\right) \\
f_{\delta}\left(x_{2}\right) \\
\vdots \\
f_{\delta}\left(x_{m-1}\right)
\end{array}\right] .
$$

The problem (23) is ill-posed in the sense that the inverse operator $C_{m}{ }^{-1}$ of $C_{m}$ exists but it is not continuous. The problem (23) has a unique solution when solving it directly will not give the right solution. Indeed, the linear operator $C_{m}$ is ill-conditioned that any numerical attempt to directly solve (23) may fail.

In order to find the stable solution from the equation (23) which is described Tikhonov regularization method. The computation of the approximate solution $\mathrm{u}_{\alpha \delta}$ consists in solving the Euler equation

$$
\mathrm{u}_{\delta}(\alpha)=\left(C_{m}^{*} C_{m}+\alpha I\right)^{-1} C_{m}^{*} f_{i}^{\delta},
$$

where $C_{m}^{*}$ is the operator adjoin to the operator $C_{m}$ and $I$ is identity matrix and $\alpha$ is regularization parameter.

To determine the regularization parameter $\alpha$ in solution $\mathrm{u}_{j}(\alpha)$ we based on the residual principle method as described in [2]. The $\alpha$ should satisfy following equation

$$
\left\|C_{m} \mathrm{u}_{\delta}\left(\alpha_{s}\right)-f_{i}^{\delta}\right\|_{L_{2}}^{2}=\delta^{2}
$$

\section{Numerical example}

Considering the problem (1-4) we need to estimate the initial temperature $\mathrm{u}_{0}(\mathrm{x})$ from given function $\mathrm{u}(\mathrm{x}, \mathrm{T})=\mathrm{f}(\mathrm{x})$ with known noise level $\delta$ where time $(\mathrm{T}=0.1$ and $\mathrm{T}=0.15)$, $(\mathrm{m}=100)$ and $\mathrm{D}=1$ for checking the approximation solution we will use the exact initial temperature $\mathrm{u}_{0}(\mathrm{x})=\sin (\mathrm{x})$ in example 1 and $\mathrm{u}_{0}(\mathrm{x})=4 \sin (3 \pi \mathrm{x})$ in example 2 . In each example, we can find $\mathrm{f}(\mathrm{x})$ function by using the equation (8) this called forward solution and add some noise to apply the inverse algorithm to estimate the initial function and check it with the exact initial temperature.

\subsection{Example 1}

Let the exact solution for the problems $(1-4)$ be

$$
\mathrm{u}_{0}(\mathrm{x})=\sin (\mathrm{x}), 0 \leq \mathrm{x} \leq 1,
$$

we consider two cases under different time $(\mathrm{T}=0.1$ and $\mathrm{T}=0.15)$ as shown in Fig. 1.

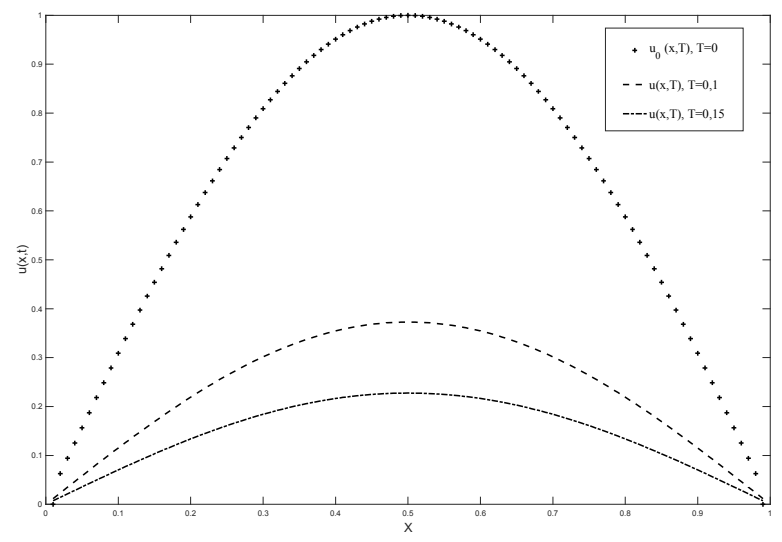

Fig. 1. Direct solution initial temperature $u_{0}(x)=\sin (x)$ 
We can add noise to each $\mathrm{u}(\mathrm{x}, \mathrm{T})$ for using them in the problem analysis. By using the equation (24) we can find estimated solutions with regularization parameters $\alpha$. We can use the set of regularization parameters to obtain the best-estimated solution $\alpha_{s}=\left\{\alpha_{1}, \alpha_{2}, \ldots, \alpha_{s}\right\}$, where $\alpha_{1}=10^{-1}, \alpha_{1}=10^{-2}$ and $\alpha_{s}=10^{-s}$ as shown in Fig. 2.

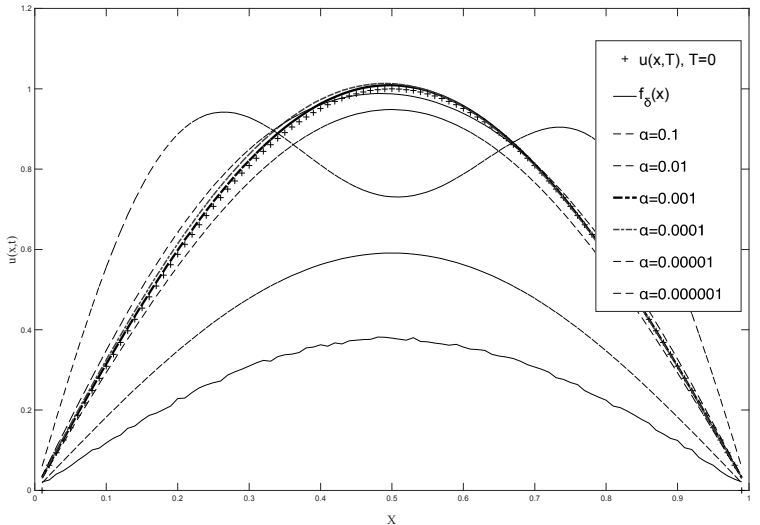

a) $\mathrm{T}=0.1$ and $\delta=0.057$

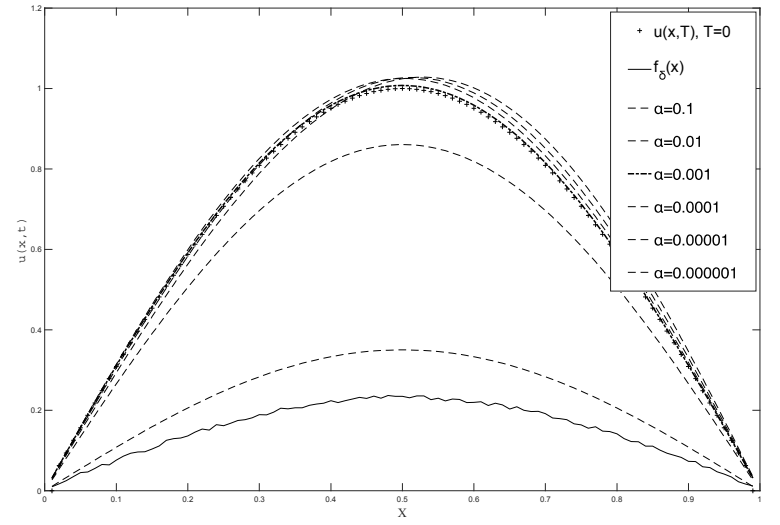

b) $\mathrm{T}=0.15$ and $\delta=0.052$

Fig. 2. Inverse solution for the initial temperature $u_{0}(x)=\sin (x)$

The best regularization parameter $\alpha$ can be selected by using the residual principle method equation (25) as shown in Tab. 1.

Table 1

Best $\alpha$ residual principle method

\begin{tabular}{|c|c|c|}
\hline$\alpha_{\mathrm{s}}$ & $\begin{array}{c}\left\|\mathrm{C}_{\mathrm{m}} \mathrm{u}_{\delta}\left(\alpha_{\mathrm{s}}\right)-\mathrm{f}_{\mathrm{i}}{ }^{\delta}\right\|_{\mathrm{L}_{2}}{ }, \\
\text { where } \mathrm{T}=0.1 \text { and } \delta=0.057\end{array}$ & $\begin{array}{c}\left\|\mathrm{C}_{\mathrm{m}} \mathrm{u}_{\delta}\left(\alpha_{\mathrm{s}}\right)-\mathrm{f}_{\mathrm{i}}{ }^{\delta}\right\|_{\mathrm{L}_{2}}{ }^{\prime} \\
\text { where } \mathrm{T}=0.15 \text { and } \delta=0.052\end{array}$ \\
\hline $1 * 10^{-1}$ & 1.121952241 & 1.089270388 \\
\hline $1 * 10^{-2}$ & 0.183193635 & 0.269894863 \\
\hline $1 * 10^{-3}$ & 0.039406461 & 0.047722743 \\
\hline $1 * 10^{-4}$ & 0.034446107 & 0.036155186 \\
\hline $1 * 10^{-5}$ & 0.034377304 & 0.036009032 \\
\hline $1 * 10^{-6}$ & 0.034269028 & 0.036003374 \\
\hline
\end{tabular}

\subsection{Example 2}

Let the exact solution for the problem (1-4) be

$$
u_{0}(x)=4 \sin (3 \pi x), 0 \leq x \leq 1,
$$

we consider two cases under different time $(\mathrm{T}=0.01$ and $\mathrm{T}=0.015)$ as shown in Fig. 3.

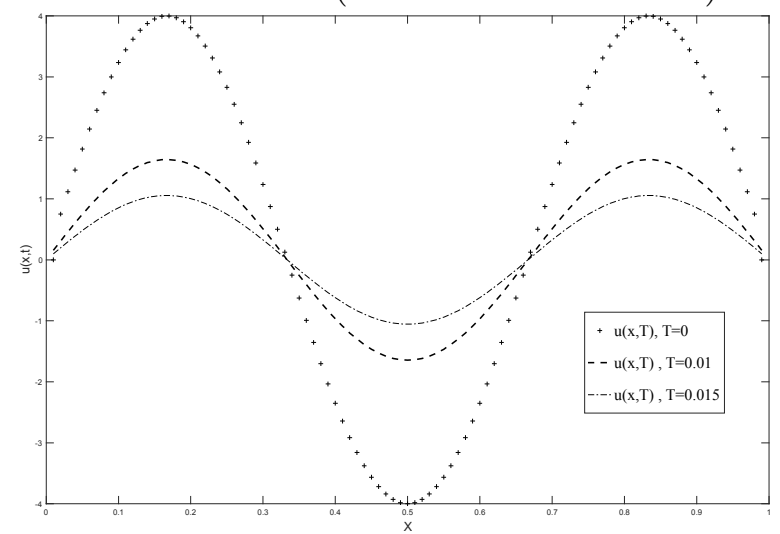

Fig. 3. Direct solution initial temperature $\mathrm{u}_{0}(\mathrm{x})=4 \sin (3 \pi \mathrm{x})$ 
In this example, we increase noise level $\delta$ and used the same set of the regularization parameters in the previous example. We obtained the best-estimated solutions as shown in Fig. 4.

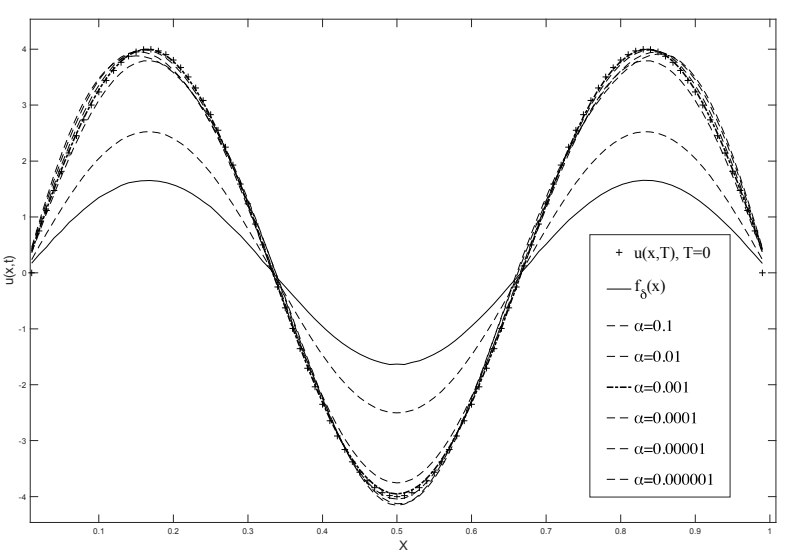

a) $\mathrm{T}=0.01$ and $\delta=0.114$

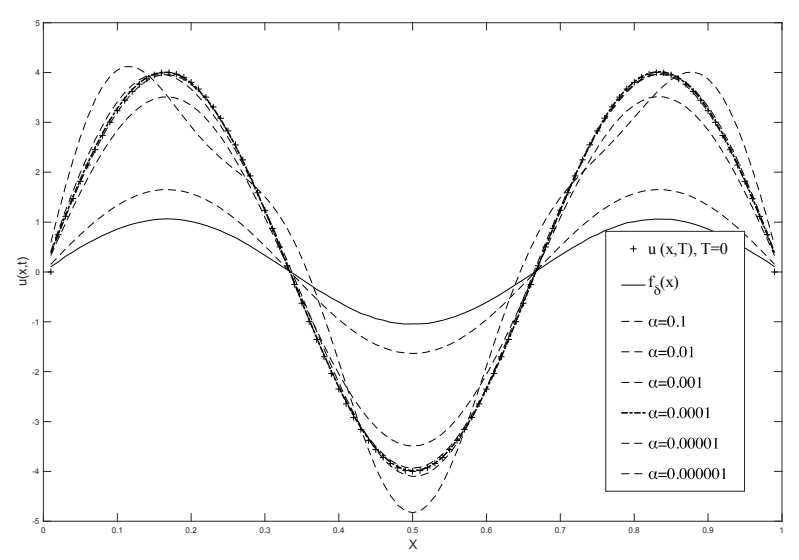

b) $\mathrm{T}=0.015$ and $\delta=0.106$

Fig. 4. Inverse solution for the initial temperature $u_{0}(x)=4 \sin (3 \pi x)$

The best regularization parameter $\alpha$ can be selected by using the residual principle method equation (25) as shown in Tab. 2.

Table 2

Best $\alpha$ residual principle method

\begin{tabular}{|c|c|c|}
\hline$\alpha_{\mathrm{s}}$ & $\begin{array}{c}\left\|\mathrm{C}_{\mathrm{m}} \mathrm{u}_{\delta}\left(\alpha_{\mathrm{s}}\right)-\mathrm{f}_{\mathrm{i}}{ }^{\delta}\right\|_{\mathrm{L}_{2}}, \\
\text { where } \mathrm{T}=0.01 \text { and } \delta=0.114\end{array}$ & $\begin{array}{c}\left\|\mathrm{C}_{\mathrm{m}} \mathrm{u}_{\delta}\left(\alpha_{\mathrm{s}}\right)-\mathrm{f}_{\mathrm{i}}{ }^{\delta}\right\|_{\mathrm{L}_{2}}, \\
\text { where } \mathrm{T}=0.015 \text { and } \delta=0.106\end{array}$ \\
\hline $1 * 10^{-1}$ & 4.32936173855166 & 4.41171841520750 \\
\hline $1 * 10^{-2}$ & 0.653083706856141 & 0.941489551857426 \\
\hline $1 * 10^{-3}$ & 0.0905050094101809 & 0.119829324858028 \\
\hline $1 * 10^{-4}$ & 0.0587797017102758 & 0.0569163570726761 \\
\hline $1 * 10^{-5}$ & 0.0578365971019751 & 0.0557491308369363 \\
\hline $1 * 10^{-6}$ & 0.0578001716547491 & 0.0550439922968124 \\
\hline
\end{tabular}

\section{Conclusion}

This work deals with the algorithm for solving the backward heat problem and some results have been collected. This problem is Cauchy ill-posed problem and special method need to solve such as problem. Fourier series method has been used by separation of variables for backward heat problem to represent the partial differential equation as Fredholm integral equation of the first kind. The numerical analyzes successfully apply to solve the inverse heat conducting problem by using discretization method to convert integral equation to a system of linear equations and using the Tikhonov's regularization method to estimate initial temperature and checking the estimated result with exact result. From the examples, we can note the algorithm was efficient to estimate the initial temperature depending on the given measurement temperature with the known noise level $\delta$. 
This paper is distributed under the terms of the Creative Commons Attribution-Non Commercial 3.0 License which permits non-commercial use, reproduction and distribution of the work without further permission provided the original work is properly cites.

\section{References}

1. Kabanikhin S.I. Inverse and Ill-posed Problems: Theory and Applications. Inverse and IllPosed Problems Series 55. De Gruyter, 2012. 24 p.

2. Tanana V.P., Sidikova A.I. Optimal Methods for Solving Ill-posed Heat Conduction Problems. Inverse and Ill-Posed Problems Series 62. De Gruyter, 2018. 40 p.

3. Duda P. Solution of Inverse Heat Conduction Problem Using the Tikhonov Regularization Method. Journal of Thermal Science. 2017. vol. 26, no. 1. pp. 60-65. DOI: 10.1007/s11630017-0910-2

4. Ahmadizadeh V., Soti Y., Pourgholi R., et al. Estimation of Heat Flux in One-dimensional Inverse Heat Conduction Problem. International Mathematical Forum. 2007. vol. 2, no. 10. pp. $455-464$.

5. Mu H., Li J., Wang X., et al. Optimization Based Inversion Method for the Inverse Heat Conduction Problems. IOP Conference Series: Earth and Environmental Science. 2017. vol. 64, no. 1. pp. 9. DOI: 10.1088/1755-1315/64/1/012094

6. Zhu Y., Liu B., Jiang P., et al. Inverse Heat Conduction Problem for Estimating Heat Flux on a Triangular Wall. Journal of Thermophys Heat Transf. 2017. vol. 31, no. 1. pp. 205-210. DOI: 10.2514/1.T4877

7. Frąckowiak A., Botkin N.D., Ciałkowski M., et al. Iterative Algorithm for Solving the Inverse Heat Conduction Problems with the Unknown Source Function. Inverse Problems in Science and Engineering. 2015. vol. 23, no. 6. pp. 1056-1071. DOI: 10.1080/17415977.2014.986723

8. Tanana V.P., Sidikova A.I. On Estimating the Error of an Approximate Solution Caused by the Discretization of an Integral Equation of the First Kind. Steklov Institute of Mathematics. 2017. vol. 299, no. 1. pp. 217-224. DOI: 10.1134/S0081543817090231 


\title{
РАЗРАБОТКА ЧИСЛЕННОГО МЕТОДА РЕШЕНИЯ ОБРАТНОЙ ЗАДАЧИ КОШИ ДЛЯ УРАВНЕНИЯ ТЕПЛОПРОВОДНОСТИ
}

\author{
(C) 2019 Х.К. Аль-Махдави \\ Южно-Уральский государственный университет \\ (454080 Челябинск, пр. им. В.И. Ленина, д. 76) \\ E-mail: hssnkd@gmail.com \\ Поступила в редакцию: 04.06.2018
}

В этой работе начальная температура была исследована в обратной задаче Коши для линейного уравнения теплопроводности, которая зависит от заданной температуры в заданное время с некоторыми шумовыми измерениями. В этой задаче начальное распределение температуры неизвестно, но вместо этого в то время известна температура, $\mathrm{t}=\mathrm{T}>0$. Задачу теплопроводности можно сформулировать так, как интегральное уравнение первого рода Фредгольма. Хорошо известно, что эта проблема является некорректной задачей, и прямое решение этой проблемы неприемлемо. Алгоритм, используемый для определения конечномерного оператора для этой задачи, также использовал метод обобщенной несоответствия для уменьшения условной проблемы вариации экстремума к безусловной проблеме изменения экстремума для интегрального уравнения. Дискретизация интегрального уравнения позволила свести эту задачу к системе линейных алгебраических уравнений. Тогда для решения аппроксимации использовался метод инверсии регуляризации Тихонова. Наконец, был представлен пример численного расчета для проверки точности оценочного решения.

Ключевые слова: некорректная задача, регуляризачия, обратная задача, теплопроводность.

\section{ОБРАЗЕЦ ЦИТИРОВАНИЯ}

Al-Mahdawi H.K. Development of a Numerical Method for Solving the Inverse Cauchy Problem for the Heat Equation // Вестник ЮУрГУ. Серия: Вычислительная математика и информатика. 2019. Т. 8, № 2. С. 22-31. DOI: 10.14529/cmse190202.

\section{Литература}

1. Kabanikhin S.I. Inverse and Ill-posed Problems: Theory and Applications. Inverse and IllPosed Problems Series 55. De Gruyter, 2012. 24 p.

2. Tanana V.P., Sidikova A.I. Optimal Methods for Solving Ill-posed Heat Conduction Problems. Inverse and Ill-Posed Problems Series 62. De Gruyter, 2018. 40 p.

3. Duda P. Solution of Inverse Heat Conduction Problem Using the Tikhonov Regularization Method // Journal of Thermal Science. 2017. Vol. 26, No. 1. P. 60-65. DOI: 10.1007/s11630-017-0910-2

4. Ahmadizadeh V., Soti Y., Pourgholi R., et al. Estimation of Heat Flux in One-dimensional Inverse Heat Conduction Problem // International Mathematical Forum. 2007. Vol. 2, No. 10. P. 455-464.

5. Mu H., Li J., Wang X., et al. Optimization Based Inversion Method for the Inverse Heat Conduction Problems // IOP Conference Series: Earth and Environmental Science. 2017. Vol. 64, No. 1. P. 9. DOI: 10.1088/1755-1315/64/1/012094

6. Zhu Y., Liu B., Jiang P., et al. Inverse Heat Conduction Problem for Estimating Heat Flux on a Triangular Wall // Journal of Thermophys Heat Transfer. 2017. Vol. 31, No. 1. P. 205-210. DOI: $10.2514 / 1 . T 4877$ 
7. Frackowiak A., Botkin N.D., Ciałkowski M., et al. Iterative Algorithm for Solving the Inverse Heat Conduction Problems with the Unknown Source Function // Inverse Problems in Science and Engineering. 2015. Vol. 23, No. 6. P. 1056-1071. DOI: $10.1080 / 17415977.2014 .986723$

8. Tanana V.P., Sidikova A.I. On Estimating the Error of an Approximate Solution Caused by the Discretization of an Integral Equation of the First Kind // Steklov Institute of Mathematics. 2017. Vol. 299, No. 1. P. 217-224. DOI: 10.1134/S0081543817090231

Аль-Махдави Хассан К. Ибрахим, аспирант, Южно-Уральский государственный университет (национальный исследовательский университет) (Челябинск, Российская Федерация) 